\title{
Extrapolation accuracy underestimates rule learning: Evidence from the function-learning paradigm
}

\author{
Nadia Said · Helen Fischer
}

Received: date / Accepted: date

\begin{abstract}
Understanding the development of non-linear processes such as economic or population growth is an important prerequisite for informed decisions in those areas. In the function-learning paradigm, people's understanding of the function rule that underlies the to-be predicted process is typically measured by means of extrapolation accuracy. Here we argue, however, that even though accurate extrapolation necessitates rule-learning, the reverse does not necessarily hold: Inaccurate extrapolation does not exclude rule-learning. Experiment 1 shows that more than one third of participants who would be classified as "exemplar-based learners" based on their extrapolation accuracy were able to identify the correct function shape and slope in a rule-selection paradigm, demonstrating accurate understanding of the function rule. Experiment 2 shows that higher proportions of rule learning than rule-application in the function learning paradigm is not due to (i) higher a priori probabilities to guess the correct rule in the rule-selection paradigm; nor is it due to (ii) a lack of simultaneous access to all function values in the function-learning paradigm. We conclude that rule application
\end{abstract}

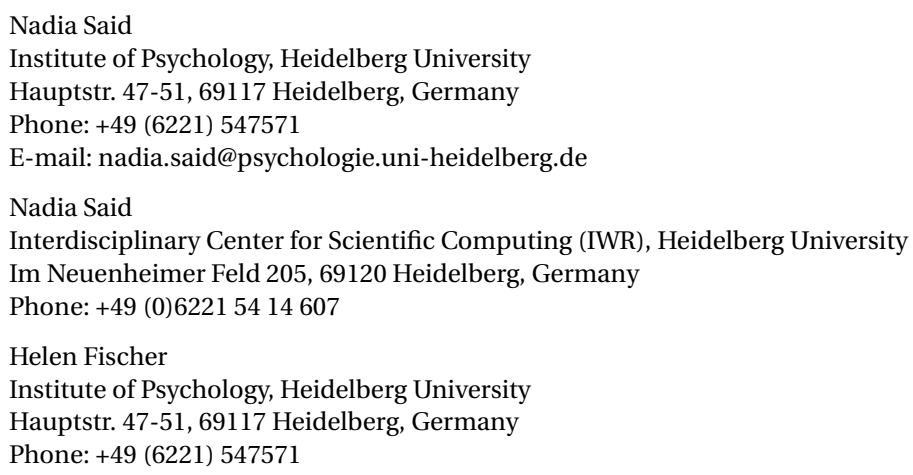


is not tantamount to rule-learning, and that assessing rule-learning via extrapolation accuracy underestimates the proportion of rule learners in function-learning experiments.

Keywords Function-learning $\cdot$ Rule-based vs exemplar-based learners $\cdot$ Non-linear processes Understanding

\section{Introduction}

Non-linear processes abound in human life, ranging from small-scale examples such as fuel consumption to large-scale, global processes such as the developments of economsubies, populations, or greenhouse gas emissions. A long-standing question in the cognitive literature is whether humans acquire an understanding of the underlying function rule when making predictions about the development of such processes. This question is often investigated in the function-learning paradigm, where participants learn about the beginning of a process with input-output pairs sampled from the underlying function, and predict the future development of that process. Typically, extrapolation accuracy, the distance between participants' predictions and the actual function values, is used to infer whether participants acquired an understanding of the function rule: It is argued that when predictions are sufficiently close to the correct function, participants must have learned the correct function rule; when predictions deviate sufficiently from the correct function, for example by showing flat extrapolations of highly non-linear processes, participants did not learn the correct function rule.

Here we argue, however, that even though sufficiently correct extrapolations necessitate previous rule learning, the reverse does not necessarily hold: Incorrect extrapolations do not exclude rulelearning. Rather, incorrect extrapolations can mirror alternative processes, such as implementation failure. Based on this theoretical argument, we investigate in how far accuracy of extrapolations coincides with rule-learning of three different exponential declining ${ }^{1}$ processes in two function-learning experiments.

In function-learning experiments, participants learn to predict continuous output (y-values) from continuous input (x-values) variables. To do so, participants are presented with an input value (for example, a time point; Fischer and Holt, 2016), and then predict the corresponding outcome value.

\footnotetext{
1 Please note that the term "exponential declining" conventionally refers to $e^{-x}$, however we will use this term throughout the paper to refer to $-e^{x}$ which is the negative of the exponential function.
} 
During training, participants receive feedback on their predictions; during test (interpolation or extrapolation), no feedback is given.

Research has shown that there are two fundamentally different types of learning style that participants may employ in function-learning experiments: Rule-based and exemplar-based learning (McDaniel et al., 2014). In exemplar-based models, participants try to memorize the given exemplars, whereas in rule-based models, participants learn the function rule underlying the to-be predicted process. Among the class of exemplar-based models, at least three different accounts exist on what participants do with the stored exemplars during extrapolation. Simple exemplar-based models, first, hold hat participants extrapolate using exemplars that are identical (or at least highly similar) to learned exemplars, thereby for example producing flat extrapolations that correspond to the stored exemplars (DeLosh et al., 1997). The Extrapolation-Association Model (EXAM; DeLosh et al., 1997), second, holds that participants retrieve the two best-matching exemplars, and extrapolate linearly through these exemplars. And the Population of Linear Experts model (POLE; Kalish et al., 2004), third, holds that participants store mappings between $\mathrm{x}$-values and matching linear functions that they retrieve for extrapolation. Rule-based models, in contrast, hold that participants use the training information provided to abstract a rule describing the ensemble of x-y pairings (Mcdaniel and Busemeyer, 2005).

While function-learning studies differ in many aspects, such as the functions used (for example Vshaped, McDaniel et al., 2014 , or periodic, Bott and Heit, 2004), the input format (entering a number, MacKinnon and Wearing, 1991, or clicking on a bar, McDaniel et al., 2014), the number of learning trials (for example 200, McDaniel et al., 2014, or 10 Fischer and Holt, 2016), and the experimental design (such as one learning, followed by one extrapolation phase, Lewandowsky et al., 2002, as opposed to several interspersed extrapolation phases, Bott and Heit, 2004), most function-learning studies have in common that extrapolation accuracy is used as a proxy for learning style. Specifically, not only is high extrapolation accuracy interpreted as signaling rule-learning, but also low extrapolation accuracy is interpreted as signaling exemplar-based or simple exemplar-based learning.

In one of the classic function-learning studies (DeLosh et al., 1997), absolute deviations of participants' extrapolations from the correct quadratic function were used to infer learning-type, and the authors concluded that flat extrapolations to a quadratic function were reflective of simple exemplarbased learning. In another experiment using quadratic functions (Lewandowsky et al., 2002), about $20 \%$ of participants were classified as being unable to learn the underlying rule based on the low fit 
of their extrapolations with the correct function. In a study with periodic functions participants were able to extrapolate (surprisingly) accurately compared to the results in other studies. The authors suggested that this difference in results may be due to participants in other experiments being unable to learn the function rule (Bott and Heit, 2004). And in a more recent study explaining individual differences in learning style, participants who showed relatively flat extrapolations to a V-shaped function were categorized as exemplar-, as opposed to rule-based learners.

The reasoning behind these studies is summarized in a theoretical argument of Kwantes and Neal (2006) who argue: "To show that you have really learned the concept, you need to demonstrate two things: You need to perform reasonably well on new items that fall within the bounds set by the training examples (so-called interpolation items), and you need to perform reasonably well on new items that fall outside the bounds set by the training examples (so-called extrapolation items)". The authors thus argue that extrapolation accuracy separates participants who learned a function rule (or "concept") from those who did not learn a function rule.

In sum, function-learning studies reviewed here share the (often implicit) assumption that provided that, participants did acquire an understanding of the correct rule, they also apply it when extrapolating. If this assumption holds, inaccurate extrapolations can indeed be interpreted as signaling the absence of rule-learning. If this assumption does not hold, however, inaccurate extrapolations are also compatible with accurate rule-learning. In other words: while accurate extrapolations are an implication of rule-learning, inaccurate extrapolations indicate learning styles other than rule-learning if, and only if, the assumption of rule application holds.

Here we put the assumption of rule-application given rule-learning to an experimental test. The reasoning behind is that participants neither need to apply a learned rule per se, nor do they need to apply it correctly. For example, participants may fail to accurately implement a learned rule, potentially because deriving extrapolation points from the abstracted rule requires substantial cognitive resources such as working memory capacity (Fischer and Holt, 2016). Also adjusting each consecutive extrapolation to previous extrapolations may be error-prone. Participants may even deliberately use comparatively simple linear extrapolations despite better knowledge.

We will use the term (a) function rule to refer to the general trend (declining), shape (exponential), and slope of a presented process. Depending on whether participants acquire an understanding of only one, two, or all three of these aspects, increasingly stricter conditions of rule-learning are 
met. We use the term (b) extrapolation style to refer to participants' extrapolations as either (1) simple exemplar-based, that is, linear extrapolation parallel to the x-axis (DeLosh et al., 1997), (2) exemplarbased, that is, linear extrapolation through the two best-matching learning points (DeLosh et al., 1997) or 3. rule-based, that is, extrapolation according to a function rule.

We report the results of two large function-learning experiments demonstrating that a substantial proportion of participants who would be classified as "exemplar-based learners" based on their extrapolation accuracy actually acquired an understanding the correct function rule. These results shed doubt on the assumption of rule-application given rule-learning. Furthermore, these results also deliver a comprehensive estimate of the extent to which rule-learning is underestimated by means of extrapolation accuracy.

\section{Experiment 1}

Experiment 1 investigated the extent to which participants who would be classified as "exemplarbased" based on their extrapolation accuracy in a classical function-learning paradigm had acquired an understanding of the function rule. To do so, participants completed two tasks: A standard functionlearning task to assess extrapolation accuracy, and a rule-selection task to assess whether participants could identify the correct function shape and slope. In the function-learning task, participants extrapolated the development of three exponential declining processes. The task consisted of one learning phase, and one extrapolation phase per process (Fischer and Holt, 2016). Participants received the instructions to extrapolate the development of different types of bacteria cultures, "Ain", "Bin", and "Cin". After the learning phase, participants completed the rule-selection task. Participants identified the function rule of the process they had just learned by selecting one of a total of six pictures displaying different function shapes and slopes.

\section{Method}

Participants. A total of 520 participants completed the experiment. Participants were recruited over MTurk, and received 1.05\$. Data from 9 participants were removed because they already participated in the pretest, thus the data from $n=511$ participants were included in the final data set. Participants 
were instructed not to use pen, paper or any other help during the study. The sample size was determined by a power analysis based on a small effect size of $r=.18$ (Fischer and Holt, 2016), $p=.05$ and $\beta=0.8$, resulting in a sample size of $n=240$ per condition.

Materials. (a) Processes. We used three variations of exponential functions based on the equation:

$$
y=1500-e^{a \cdot(x+50)+2}
$$

with $a=[0.045,0.040,0.046]$. In the following we refer to the function with $a_{1}=0.045$ as process Ain, with $a_{2}=0.040$ as Bin, and with $a_{3}=0.046$ as Cin.

Exponential declining functions were chosen because they represent a very difficult function to extrapolate (Busemeyer et al., 1997), and hence because of their strong deviation from the "cognitive default" of positive linearity, a particularly strong case for rule-learning can be made (DeLosh et al., 1997; Kalish et al., 2004; Kwantes and Neal, 2006).

(b) Rule-selection task. Participants were presented with three graphs, displaying three different function shapes of two different slopes each: two linearly decreasing functions, two exponential declining functions, and two Gaussian functions. Participants were asked to indicate, "Which graph describes the shape of the development of the bacteria best?". Participants entered the number of the graph into a text box (Figure 1).

Linear functions were chosen as they represent the most basic and frequently found extrapolation style (Carroll, 1963; Busemeyer et al., 1997) that is furthermore employed in exemplar-based, as well as simple exemplar-based extrapolations; Gaussian functions were chosen to assess whether participants believed the process to be non-monotonical; and the exponential declining functions were chosen to assess whether participants could correctly identify the correct function shape, and potentially also slope. The slopes displayed were $0.045_{\text {correct }}$ and 0.040 for Ain, $0.040_{\text {correct }}$ and 0.046 for Bin, and $0.046_{\text {correct }}$ and 0.043 for Cin. Slopes for all functions were chosen in a way such that $y$-values of functions remained between 0 and 1500 .

Procedure. Each participant extrapolated 3 processes. Each process consisted of 13 trials, 8 learning and 5 extrapolation trials. At the beginning of each process, participants were given the starting point of that process, that is, the number of bacteria at time point 0 (1430 for Ain, 1445 for Bin, and 1426 for Cin). Processes were shown in a fixed order. During each trial, participants were shown the 
Which graph describes the shape of the development of the bacteria best?

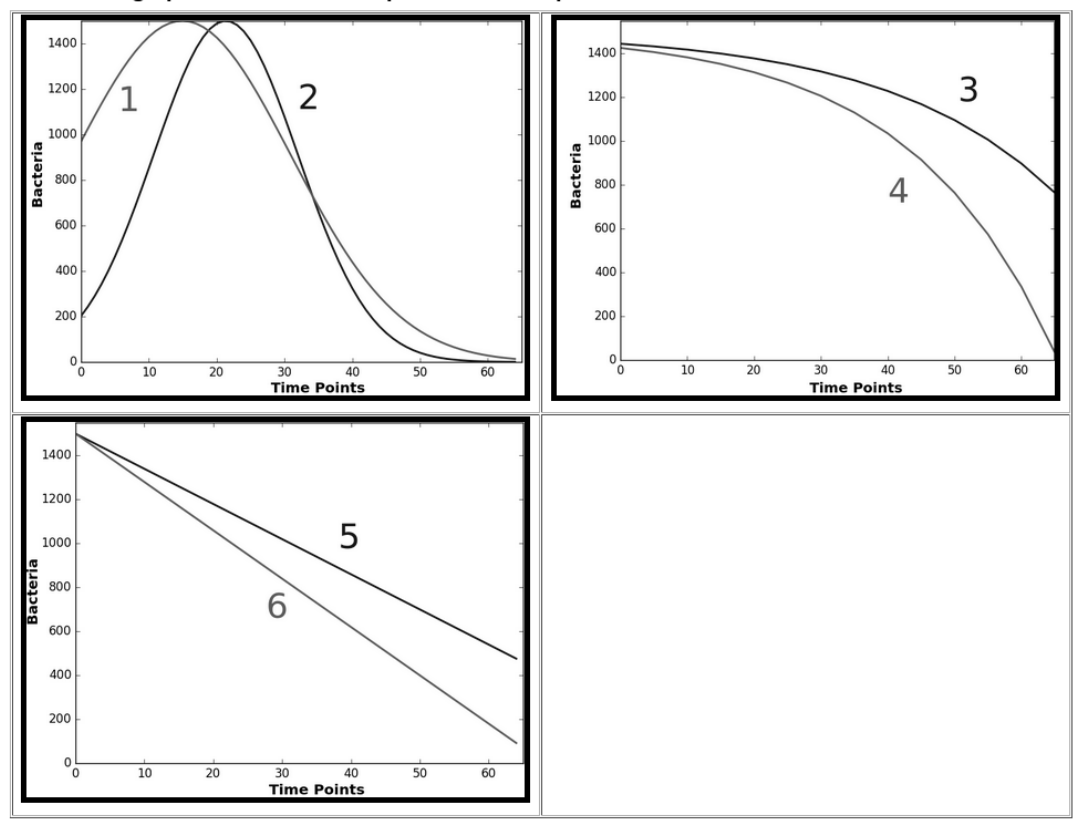

Please enter

the graph

Fig. 1 Example of rule-selection task for process Bin. The figure displays the functions given to participants with the instructions to choose the process they have just learned (Correct: Function no. 3.)

current time point and predicted the number of bacteria for that time point by entering their extrapolation as a number into a text box ("I guess the number of bacteria is ..."). During the learning phase, participants received feedback in terms of the correct number of bacteria for each time point, immediately after entering their extrapolation (“You guessed: ... . Actual number: ...”).

To control for the effect of seeing different function shapes in the rule-selection task on extrapolation accuracy, participants were randomly allocated to complete the rule-selection task immediately before, or immediately after the extrapolation phase. 


\section{Results}

Dependent variables. To measure extrapolation accuracy in the function-learning task, the relative root mean square error (rRMSE) was used :

$$
\operatorname{rRMSE}=\sqrt{\frac{1}{n} \cdot \sum_{i=1}^{n}\left(\frac{\left(y_{i}-z_{i}\right)}{z_{i}}\right)^{2}}
$$

with $y_{i}$ : extrapolation and $z_{i}$ : correct function value.

To measure understanding of the function rule, the rule selection task distinguished between identifying the correct function shape, that is, choosing either of the two exponential functions; and additionally identifying the correct function slope, that is, choosing the exponential function AND the correct slope.

Outliers. We excluded individual extrapolations more than five standard deviations above or below the mean of each time point $(0.51 \%$ of the total number of extrapolations). If more than two out of the five extrapolation trials were excluded, the process was treated as missing for this participant (Fischer and Holt, 2016). In total, for 4 participants processes were excluded, resulting in 509 participants for process Ain, and 510 participants for processes Bin and Cin.

Order of rule-selection and extrapolation. In order to assess whether showing participants pictures of the correct function impacted extrapolation accuracy, we compared extrapolation accuracy in the group completing the rule-selection task before $(M=3.52, S D=2.07)$ versus after $(M=3.55$, $S D=2.02)$ the extrapolation phase. Accuracy was marginally but not significantly higher in the group performing the rule-selection task before the extrapolation phase, $F(3,503)=2.36, p=.07$, Pillais' Trace $=0.014$. Thus, in the following, results for both groups are presented together.

Extrapolation accuracy by function slope. We assessed whether extrapolation accuracy varied by function slope. As the assumptions of homogeneity of variances $(F(2,1526)=324.12, p<.001)$ as well as of normality ( $W=0.69, p<.001$ ) were not met, Kruskal-Wallis rank sum tests were conducted. Results showed that extrapolation accuracy differed between the three processes as a function of slope $\chi^{2}(2)=1204.3, p<.001$ in that prediction accuracy was higher for $\operatorname{Bin}\left(M_{B i n}=0.30, S D_{B i n}=1.00\right)$ compared to Ain $\left(M_{A i n}=1.50, S D_{\text {Ain }}=1.12\right) z=17.82$, each $p<.001$. Interestingly, the drop in accuracy was particularly steep from Bin to the steepest function Cin $\left(M_{C i n}=8.86, S D_{C i n}=5.4\right)$ 
$z=-34.70, p<.001$. These results are in line with previous findings that participants have a tendency towards linear extrapolation, and hence extrapolation accuracy decreases as function slope increases.

Proportion of participants per extrapolation style. Participants' extrapolation styles were categorized based on their extrapolation accuracy (McDaniel et al., 2014). Specifically, we determined the deviation (rRMSE) of each participant's extrapolation accuracy including a 95\% confidence interval from these three cases: (1) $\mathbf{r R M S E}_{\text {Exp }}$ : The deviation from the correct function, (2) $\mathbf{r R M S E}_{\text {LinSlope0 }}$ : the deviation from a linear extrapolation with slope 0 through the last learning point, (3) $\mathbf{r R M S E}_{\text {Lin: }}$ the deviation from a linear extrapolation through the last two learning points. Confidence intervals were calculated as follows:

$$
\mathrm{CI}_{ \pm}=\bar{y}_{i} \pm 2.776 \cdot \frac{\sigma_{i}}{\sqrt{n}}
$$

with $\overline{y_{i}}$ :rRMSE for each time point i.

Extrapolations were categorized into the different groups based on whether their entire $\mathrm{CI}_{ \pm}$was (a) above rRMSE $_{\text {LinSlope0 }}$, indicating extrapolation parallel to the $\mathrm{x}$-axis (simple exemplar-based extrapolation), (b) below $\mathbf{r R M S E}_{\text {LinSlope } 0}$ but above $\mathbf{r R M S E}_{\text {Lin }}$, indicating linear extrapolation through the last two learning time points (exemplar-based extrapolation); or (c) below $\mathbf{r R M S E}_{\mathbf{L i n}}$, indicating the most accurate extrapolation (rule-based extrapolation). Figure 2 displays an exemplatory categorization for process Bin.

Relationship between rule-learning and rule-based extrapolation. In order to investigate the relationship between rule-learning and extrapolation style, we determined the association between rule selection and extrapolation style in logistic regressions, separately for each of the three processes, Ain, Bin, and Cin. We distinguished between (i) choosing the correct function shape, and (ii) choosing the correct function shape AND slope. Choosing the correct function shape, (i), was significantly related to extrapolation style for each process, $\chi_{\text {Ain }}^{2}(1)=11.13, \chi_{\text {Bin }}^{2}(1)=14.86$, and $\chi_{\text {Cin }}^{2}(1)=13.73$, each $p<.001$, suggesting that, unsurprisingly, learning of the correct function shape was related to rulebased extrapolations. Choosing the correct function shape AND slope was associated with extrapolation style for processes Ain and Cin, $\chi_{\text {Ain }}^{2}(1)=36.93, \chi_{\text {Cin }}^{2}(1)=16.83, p<.001$, but not for process Bin, $\chi_{\mathrm{Bin}}^{2}(1)=1.11, p=.29$, suggesting that learning of the correct function slope was related to rule-based extrapolations, except for extrapolation of the process with the lowest slope. 

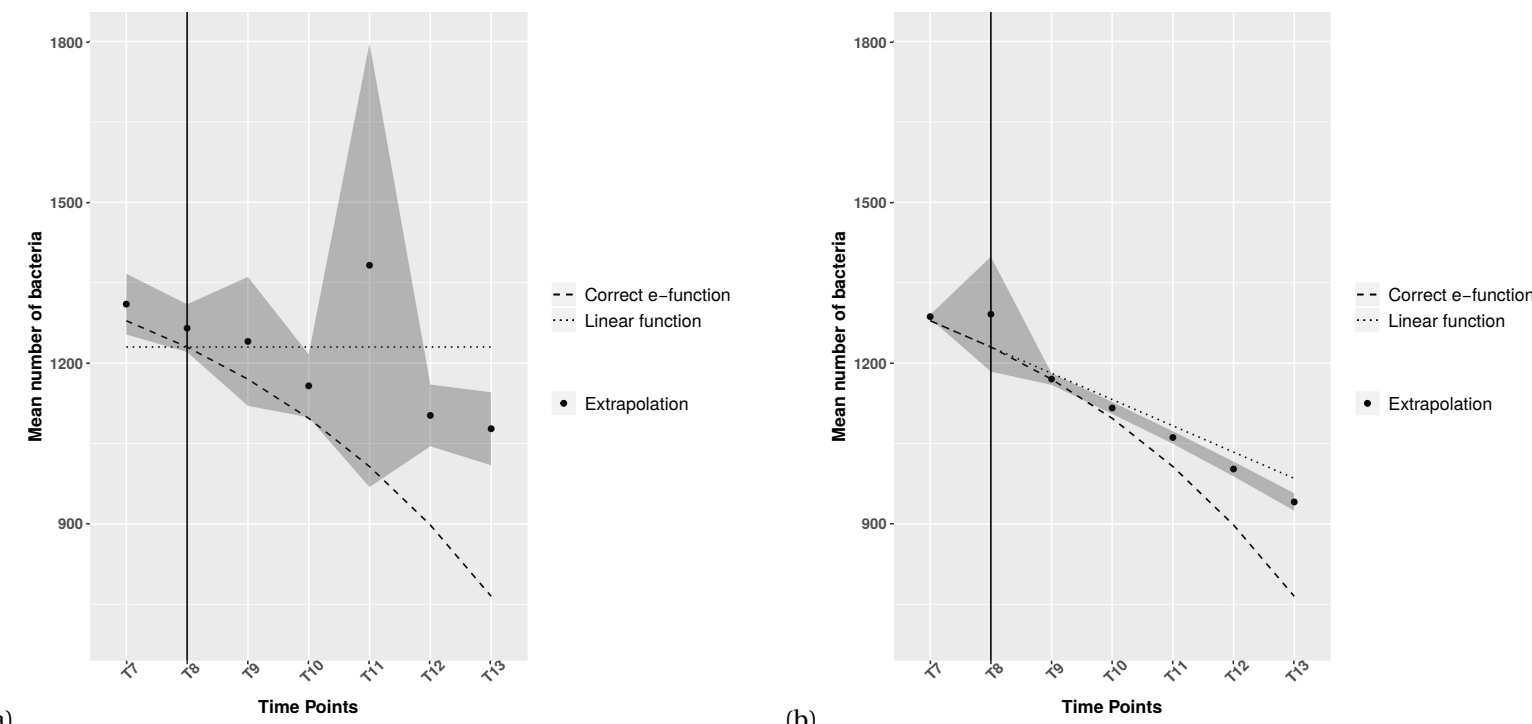

(a)

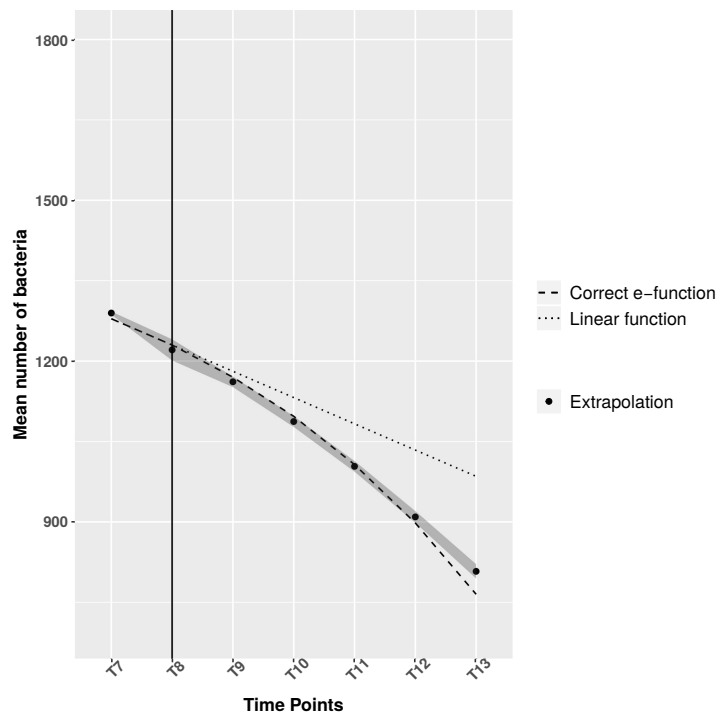

(c)

Fig. 2 Example of extrapolations for process Bin. The figure displays the number of bacteria predicted by participants who were classified as (a) simple-exemplar based , (b) exemplar-based and (c) rule-based learners based on their extrapolation accuracy. Dark-grey area: $95 \%$ confidence band.

To test the extent to which exemplar-based, or simple exemplar-based extrapolation styles exclude rule-learning, Table 1 displays the proportion of participants who could correctly identify the correct function shape, separately for each extrapolation style. In the group displaying simple exemplar-based extrapolation, $46 \%$ of participants were able to identify the correct function shape, while only $31 \%$ of 
participants estimated the function was actually linear. A similar pattern held for the group displaying exemplar-based extrapolation, where $61 \%$ of participants chose the correct function shape, and only $24 \%$ estimated the function to be linear. For the group displaying rule-based extrapolation, $65 \%$ chose the correct function shape. In sum, the relative majority of participants displaying simple exemplarbased extrapolations, and even the absolute majority of participants displaying exemplar-based extrapolations could identify the correct function shape as exponential declining.

Table 1 Proportion of participants selecting one of the three function shapes in the rule-selection task, per extrapolation style.

\begin{tabular}{llllcl}
\hline \hline Style & Process & Gaussian & Linear & Exponential & $\chi^{2}$-test \\
\hline Simple & Ain & $59(21 \%)$ & $73(27 \%)$ & $144(52 \%)$ & $\chi^{2}(2, N=276)=45.15^{* * *}$ \\
exemplar- & Bin & $59(28 \%)$ & $45(22 \%)$ & $104(50 \%)$ & $\chi^{2}(2, N=208)=27.41^{* * *}$ \\
based & Cin & $49(18 \%)$ & $127(47 \%)$ & $97(35 \%)$ & $\chi^{2}(2, N=273)=34.02^{* * *}$ \\
\hline \multirow{2}{*}{$\begin{array}{llllc}\text { Exemplar- } \\
\text { based }\end{array}$} & Total & $\mathbf{2 2 \%}$ & $\mathbf{3 2 \%}$ & $\mathbf{4 6 \%}$ & \\
\hline & Ain & $19(14 \%)$ & $26(19 \%)$ & $93(67 \%)$ & $\chi^{2}(2, N=138)=72.57^{* * *}$ \\
& Cin & $36(18 \%)$ & $41(20 \%)$ & $125(62 \%)$ & $\chi^{2}(2, N=202)=74.27^{* * *}$ \\
& Total & $\mathbf{1 5 \%}$ & $\mathbf{2 4 \%}$ & $\mathbf{6 1 \%}$ & \\
Rule-based & Ain & $16(17 \%)$ & $14(15 \%)$ & $65(68 \%)$ & $\chi^{2}(2, N=95)=52.70^{* * *}$ \\
& Bin & $16(16 \%)$ & $12(12 \%)$ & $72(72 \%)$ & $\chi^{2}(2, N=100)=67.52^{* * *}$ \\
& Cin & $15(22 \%)$ & $17(24 \%)$ & $38(54 \%)$ & $\chi^{2}(2, N=70)=13.91^{* * *}$ \\
\hline
\end{tabular}

In total, the proportion of participants who had acquired an understanding of the correct function rule in the learning phase (as indicated by the rule-selection task) but did not apply this in the extrapolation phase (as indicated by classifications of their extrapolation style based on extrapolation accuracy) was $47 \%$ for process Ain, $45 \%$ for Bin, and $36 \%$ for Cin (Table 2).

Table 2 Proportion of participants identifying the correct exponential function shape, and applying exemplar-based and simple exemplar-based extrapolations, per process (Ain, Bin, and Cin).

\begin{tabular}{lll}
\hline \hline Style & Process & Exponential \\
\hline \multirow{2}{*}{ Simple-\& } & Ain & $237(47 \%)$ \\
Exemplar-based & Bin & $229(45 \%)$ \\
& Cin & $185(36 \%)$ \\
\hline
\end{tabular}


As the stricter criterion of rule-learning, (ii) we determined the number of participants choosing not only the correct function shape but also function slope, per extrapolation style. As Table 3 shows, the proportion of participants choosing the correct function slope increased with extrapolation style, from simple exemplar-based, to exemplar-based, to rule-based. Across all three processes, $24 \%$ of participants displaying simple-exemplar-based extrapolations, and $37 \%$ of participants displaying exemplar-based extrapolations, were able to identify the correct function slope. These results suggest that even among those participants who had acquired a deep understanding of the function rule in that they could identify the correct shape AND slope, a considerable proportion of participants did not apply this understanding when extrapolating, but rather used exemplar-based, or even simple exemplar-based extrapolation styles.

The last column of Table 3 displays the proportion of participants who could identify the correct slope, out of those who could identify the correct shape. Results show that while for processes Bin and Cin, around half of participants who could identify the correct shape also identified the correct slope, results were different for the steepest process Cin in that the vast majority of participants who identified the correct shape also identified the correct slope.

Table 3 Proportion of participants selecting the correct function shape AND slope, per extrapolation style.

\begin{tabular}{|c|c|c|c|c|}
\hline Style & Process & Correct shape AND slope & Total & $\frac{\text { Correct shape AND slope }}{\text { Correct shape }} \cdot 100$ \\
\hline \multirow{3}{*}{$\begin{array}{l}\text { Simple } \\
\text { exemplar- } \\
\text { based }\end{array}$} & Ain & $44(16 \%))$ & \multirow{3}{*}{$24 \%$} & $31 \%$ \\
\hline & Bin & $65(31 \%)$ & & $63 \%$ \\
\hline & Cin & $70(26 \%)$ & & $72 \%$ \\
\hline \multirow{3}{*}{$\begin{array}{l}\text { Exemplar- } \\
\text { based }\end{array}$} & Ain & $51(37 \%))$ & \multirow{3}{*}{$\mathbf{3 7 \%}$} & $55 \%$ \\
\hline & Bin & $76(38 \%)\}$ & & $61 \%$ \\
\hline & Cin & $62(37 \%)$ & & $70 \%$ \\
\hline \multirow{3}{*}{ Rule-based } & Ain & $36(45 \%))$ & \multirow{3}{*}{$44 \%$} & $55 \%$ \\
\hline & Bin & $50(36 \%)\}$ & & $69 \%$ \\
\hline & Cin & $35(50 \%)$ & & $92 \%$ \\
\hline
\end{tabular}

Interestingly, for all three extrapolation styles, the proportion of participants who could correctly identify the correct function shape dropped $15 \%$ for the steepest function Cin compared to the proportion of participants who could correctly identify the correct function shape for processes Ain and 
Bin. This result contrasts results on extrapolation accuracy for Cin $\left(M_{\operatorname{Cin}}=8.86\right)$ which dropped by $83 \%$ compared to Ain $\left(M_{A i n}=1.50\right)$, and even $97 \%$ compared to Bin $\left(M_{B i n}=0.30\right)$.

Prevalence of rule-learning based on rule-selection task vs. extrapolation accuracy. Table 4 compares the proportion of participants who would be classified as rule-learners, based on accuracy in the rule-selection task as opposed to extrapolation accuracy. Results show that while the minority $\left(<20 \%, \chi^{2}(2, N=1529)=238, p<.001\right)$ of participants would be classified as rule-learners based on extrapolation accuracy, the relative majority of participants could identify the correct function shape $\left(>50 \%, \chi^{2}(2, N=1529)=308, p<.001\right)$, and even slope $\left(>25 \%, \chi^{2}(3, N=1529)=53.33, p<.001\right)$.

Table 4 Proportion of participants classified as rule-based, exemplar-based or simple exemplar-based learners in the functionlearning and rule-selection paradigm, per process (Ain, Bin and Cin).

\begin{tabular}{|c|c|c|c|c|c|c|c|}
\hline & \multicolumn{3}{|c|}{ Traditional FL paradigm } & \multicolumn{4}{|c|}{ Rule-selection paradigm } \\
\hline & $\begin{array}{l}\text { Simple } \\
\text { exemplar- } \\
\text { based }\end{array}$ & $\begin{array}{l}\text { Exemplar- } \\
\text { based }\end{array}$ & Rule-based & Gaussian & Linear & $\begin{array}{l}\text { Exponential } \\
\text { (shape) }\end{array}$ & $\begin{array}{l}\text { Exponential } \\
\text { (slope) }\end{array}$ \\
\hline Ain & 276 (54\%) & $138(27 \%)$ & 95 (19\%) & 94 (19\%) & $113(22 \%)$ & 302 (59\%) & $138(27 \%)$ \\
\hline Bin & $208(41 \%)$ & $202(40 \%)$ & $100(19 \%)$ & $111(22 \%)$ & 98 (19\%) & 301 (59\%) & 177 (35\%) \\
\hline Cin & $273(53 \%)$ & $167(33 \%)$ & $70(14 \%)$ & $87(17 \%)$ & $200(39 \%)$ & $223(44 \%)$ & $167(33 \%)$ \\
\hline Total & $50 \%$ & $33 \%$ & $17 \%$ & $19 \%$ & $27 \%$ & $54 \%$ & $32 \%$ \\
\hline
\end{tabular}

\section{Summary 1}

Experiment 1 showed that a substantial proportion of participants who had acquired an understanding of the correct function rule in the learning phase of a function-learning experiment (as indicated by the rule-selection task) did not apply their understanding in the extrapolation phase (as indicated by classifications of their extrapolation style based on extrapolation accuracy). Specifically, out of those participants who would be classified as exemplar-based learners based on their extrapolation accuracy, $61 \%$ were able to accurately identify the correct function shape, and $37 \%$ were able to identify the correct function shape AND slope. Moreover, only $32 \%$ of participants showing simple exemplar-based, and $24 \%$ of participants showing exemplar-based extrapolations believed the functions to be actually linear. These results suggest that (i) extrapolation accuracy underestimates rulelearning in the classical function-learning paradigm, and that (ii) up to certain extent, participants are aware of the non-linearity of the process, even if their extrapolations suggest otherwise. 
However, there are two limitations to Experiment 1. First, even though selection of the exponential shape was clearly above guessing rate in all three extrapolation styles, a priori probabilities to guess correctly were considerably higher in the rule-selection task compared to extrapolation in the standard function-learning task. To address this limitation, Experiment 2 required participants to indicate their understanding of the function shape not by selecting a picture, but by drawing their understanding of the function shape into a grid. And second, it remains unclear why participants fail to apply their rule understanding in the classical function-learning paradigm. One plausible explanation could be that participants make implementation errors in the classical function-learning paradigm where $\mathrm{x}-\mathrm{y}$ pairings are given only consecutively, whereas in the rule-selection task participants have simultaneous access to all function values. To address this second limitation, Experiment 2 introduced another control condition, where all extrapolations were displayed on the same page for a given process, so that current as well as all previous extrapolations were visible to participants.

\section{Experiment 2}

Experiment 1 showed that a substantial proportion of exemplar-based or even simple exemplar-based extrapolators had acquired an accurate understanding of the function rule, indicating that the number of rule-learning was underestimated previously by using extrapolation accuracy as a proxy for rulelearning. Experiment 2 provided equal a priori probabilities between a standard function-learning condition and an alternative paradigm in which participants indicated their understanding of the process by drawing the function into a grid (grid condition). Furthermore, we added a third condition in which participants' extrapolations were displayed on one screen instead of in consecutive order (summary function-learning condition).

\section{Method}

Participants. A total of 918 MTurk participants completed the experiment. Sample size was determined by computing a power analysis based on $f=.1, p=.05$ and $\beta=.8$, resulting in a sample size of $n=323$ per condition. Data from 176 participants were removed because inspection of MTurk IDs revealed participants had taken part in either Experiment 1, or a pretest. Participants were instructed 
not to use pen, paper or a calculator during the study. We included a statement at the end of the study in which participants had to confirm that they did not do so ("I confirm that I did NOT use a calculator, pen or paper"). A total of 15 participants were excluded because they reported having used aids. Additionally, in order for the grid condition to be fully comparable with the function-learning conditions, we checked for the position (one click per time point) and order (clicks starting from time point T1 followed by T2 and so on) of extrapolations. Out of the 232 participants in the grid condition, 67 were excluded because they violated this requirement. A total of $\mathrm{N}=660$ participants were included in the final data set.

Materials. Two of the processes from Experiment 1 were used, the development of the bacteria cultures Ain and Bin.

Procedure. Participants were randomly assigned to three groups: (a) A standard function-learning condition identical to Experiment 1 that served as a baseline. Participants entered their extrapolations as numbers, and extrapolations were displayed sequentially; (b) A grid condition where participants drew the function shape by clicking the respective positions on a grid; and (c) A summary functionlearning condition where participants entered their extrapolations in numbers and all time points were displayed as value-pairs on one screen, so that participants were able to see all previous function values (Figure 3). To ensure comparability between the three conditions, the maximum and minimum extrapolations were restricted to values $0-1550$, in steps of 1 ; and the clicks on the grid were restricted to the same number as the entries in both function-learning conditions.

\section{Results}

Dependent variables. Calculation of extrapolation accuracy (rRMSE) in the two function-learning conditions was identical to Experiment 1. We applied the same procedure regarding outliers as in Experiment 1 ( $0.11 \%$ of the total number of extrapolations).

To assess rule-learning in the grid condition, we used two types of approaches:

(1) Calculating the first derivatives. Calculating the first derivatives allows us to determine whether participants extrapolated (a) linearly through the last two learning points (exemplar-based extrapolation), or (b) according to the function rule (rule-based extrapolation) (McDaniel et al., 2014). This is because in case of (a), derivatives must be constant, whereas in case of (b), derivatives must be strictly 


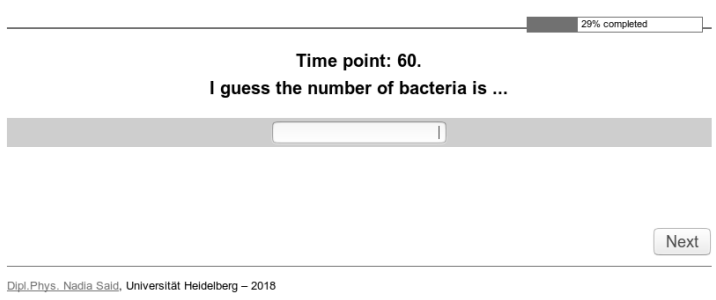

(a)

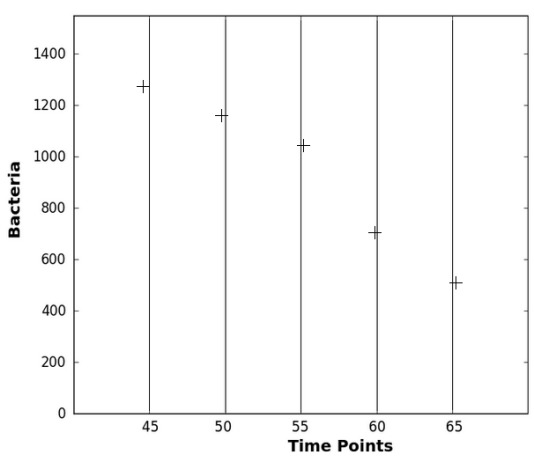

(b)

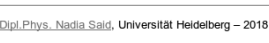

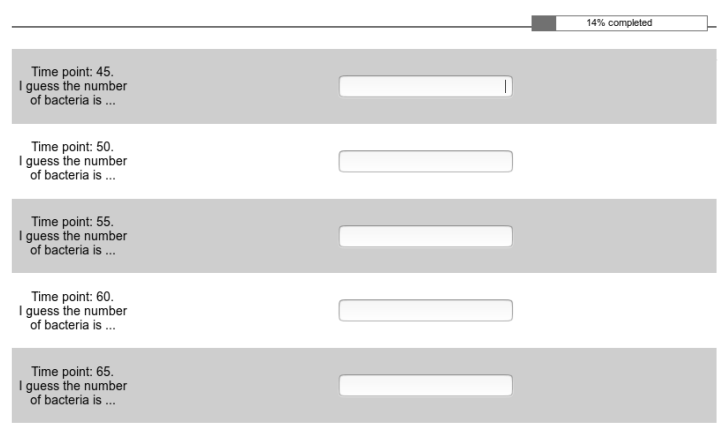

(c) Dipl Phys. Nadia Sald, Universitiat Heideloerg - 2018

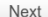

Fig. 3 Screenshots of the three conditions. The figure displays the three conditions: (a) standard function learning, (b) grid, and (c) summary function-learning. Valid numbers participants could enter: $0-1550$.

monotonic decreasing as $\frac{d}{d x}\left(-e^{x}\right)=-e^{x}$ with the negative sign reflecting the trend of the process. This allows us to evaluate whether participants abstracted a rule about the exponentiality of the process in that it is increasingly declining. To do so, we used three different approaches varying in strictness of what counts as strictly decreasing derivatives: (1) slopes of the lines through the first and the second, as well as the first and the last extrapolation point were strictly decreasing; (2) slopes through the first and two other extrapolation points have to be strictly decreasing; and (3) slopes though the first and all other extrapolation points have to be strictly decreasing. Please note that regarding the first criterion (1) other non-linear functions could also meet this condition. However, it is save to say that regarding (1) participants have at least understood that the process is not linear. 
(2) Least squares approach. Using a least squares approach, we classified the functions drawn in the grid condition as rule-based, exemplar-based, or non-distinguishable based on the deviation (RMSE) of clicks on the grid from three models: If

$$
\mathrm{RMSE}_{\text {linear }}>\mathrm{RMSE}_{\text {exponential }}+\mathrm{RMSE}_{\text {linear }} * 25 \% \text {, }
$$

participants were classified as rule-based learners; if

$$
\mathrm{RMSE}_{\text {linear }}<\mathrm{RMSE}_{\text {exponential }}-\mathrm{RMSE}_{\text {linear }} * 25 \% \text {, }
$$

participants were classified as exemplar-based learners; for all other cases, participants were classified as non-distinguishable. To ensure comparability between the models, only the slope parameter $a$ was allowed to vary, and all other parameters were fixed.

Extrapolation accuracy in standard versus summary function-learning. To assess whether comparatively low proportions of (accurate) rule-application in the classical function-learning paradigm were due to a lack of simultaneous access to all previous function values, we compared extrapolation accuracy in (a) the standard function-learning, with (b) the summary function-learning condition. Results showed that extrapolation accuracy was not higher in the summary $\left(M_{\operatorname{Ain}_{1}}=1.54, S D_{\operatorname{Ain}_{1}}=0.80\right.$, $\left.M_{\mathrm{Bin}_{1}}=0.27, S D_{\mathrm{Bin}_{1}}=0.19\right)$, compared to the standard function-learning condition $\left(M_{\mathrm{Ain}_{2}}=1.53\right.$, $\left.S D_{\mathrm{Ain}_{2}}=0.84, M_{\mathrm{Bin}_{2}}=0.26, S D_{\mathrm{Bin}_{2}}=0.19\right), F(2,492)=0.027, p=.97$, Pillais' Trace $=0.0001$. This result suggests that having access to all function values did not increase rule application per se, nor did it increase the accuracy of rule-application.

Proportion of rule-based extrapolation in the two function-learning conditions. For conditions (a) standard function-learning and (b) summary function-learning, participants' extrapolation styles were classified based on extrapolation accuracy. Table 5 shows that for both function-learning conditions, between 19\% (Ain) and 32\% (Bin) were classified as rule-based extrapolators. 
Table 5 Proportion of participants classified as showing each of the three extrapolation styles based on extrapolation accuracy, per processes (Ain and Bin).

\begin{tabular}{|c|c|c|c|c|c|c|}
\hline & \multicolumn{2}{|c|}{ Simple exemplar-based } & \multicolumn{2}{|c|}{ Exemplar-based } & \multicolumn{2}{|c|}{ Rule-based } \\
\hline & Standard FL & Summary FL & Standard FL & Summary FL & Standard FL & Summary FL \\
\hline & $148(60 \%)$ & $139(56 \%)$ & $58(23 \%)$ & 58 (24\%) & $42(17 \%)$ & $50(20 \%)$ \\
\hline Total Ain & \multicolumn{2}{|c|}{$58 \%$} & \multicolumn{2}{|c|}{$23 \%$} & \multicolumn{2}{|c|}{$19 \%$} \\
\hline & $79(32 \%)$ & $72(29 \%)$ & $87(35 \%)$ & $99(40 \%)$ & $82(33 \%)$ & $76(31 \%)$ \\
\hline Total Bin & \multicolumn{2}{|c|}{$30 \%$} & \multicolumn{2}{|c|}{$38 \%$} & \multicolumn{2}{|c|}{$32 \%$} \\
\hline
\end{tabular}

Proportion of rule-learning in the grid condition. (1) Calculating the first derivatives. We calculated the first derivatives $\left(d_{1,2}, d_{1,3}, d_{1,4}, d_{1,5}\right)$ by calculating the slopes between the first extrapolation point and the following 4 points. Participants were classified as having understood the function rule as being non-linear if (a) they captured the trend of the process $\left(d_{1, j}<0, \forall j \in\{2,3,4,5\}\right)$ and (b) if slopes of the lines through the first and the second, as well as the first and the last extrapolation point were strictly monotonic decreasing $\left(d_{1,5}<d_{1,2}\right)$. Following that classification, $48 \%$ of participants had abstracted the function rule for process Ain, and $56 \%$ for process Bin (Figure 4). We employed the same method for the next stricter criterion (three out of the four slopes), resulting in $28 \%$ of participants having abstracted the function rule for process Ain, and 39\% of participants having abstracted the function rule for process Bin. Only when using the strictest possible criterion where the values for all four slopes have to decrease strictly monotonically, results were comparable to those based on extrapolation accuracy in that $20 \%$ of the participants were classified as having abstracted the function rule for process Ain, and 29\% for process Bin (Table 6).

Table 6 Proportion of participants classified as "rule-based learners" in the derivatives approach.

\begin{tabular}{cccc}
\hline \hline & $d_{1,5}<d_{1,2}$ & $d_{1,5}<d_{1,3}<d_{1,2}$ or $d_{1,5}<d_{1,4}<d_{1,2}$ & $d_{1,5}<d_{1,4}<d_{1,3}<d_{1,2}$ \\
\hline Ain & $80(48 \%)$ & $92(28 \%)$ & $33(20 \%)$ \\
Bin & $93(56 \%)$ & $128(39 \%)$ & $48(29 \%)$ \\
\hline
\end{tabular}

and $d_{1, j}<0, \forall j \in\{2,3,4,5\}$ for all four classification criterions.

\begin{tabular}{llll}
\hline$\chi_{\text {Ain }}^{2}$ & $\chi^{2}(1)=55.87^{* * *}$ & $\chi^{2}(1)=9.33^{* *}$ & $\chi^{2}(1)=0.08, p=.77$ \\
$\chi_{\text {Bin }}^{2}$ & $\chi^{2}(1)=30.35^{* * *}$ & $\chi^{2}(1)=3.83, p=.05$ & $\chi^{2}(1)=0.34, p=.56$ \\
\hline
\end{tabular}

Comparison of proportions FL (standard \& summary) and derivatives approach, ${ }^{* *} p \leq .01,{ }^{* * *} p \leq .001$. 

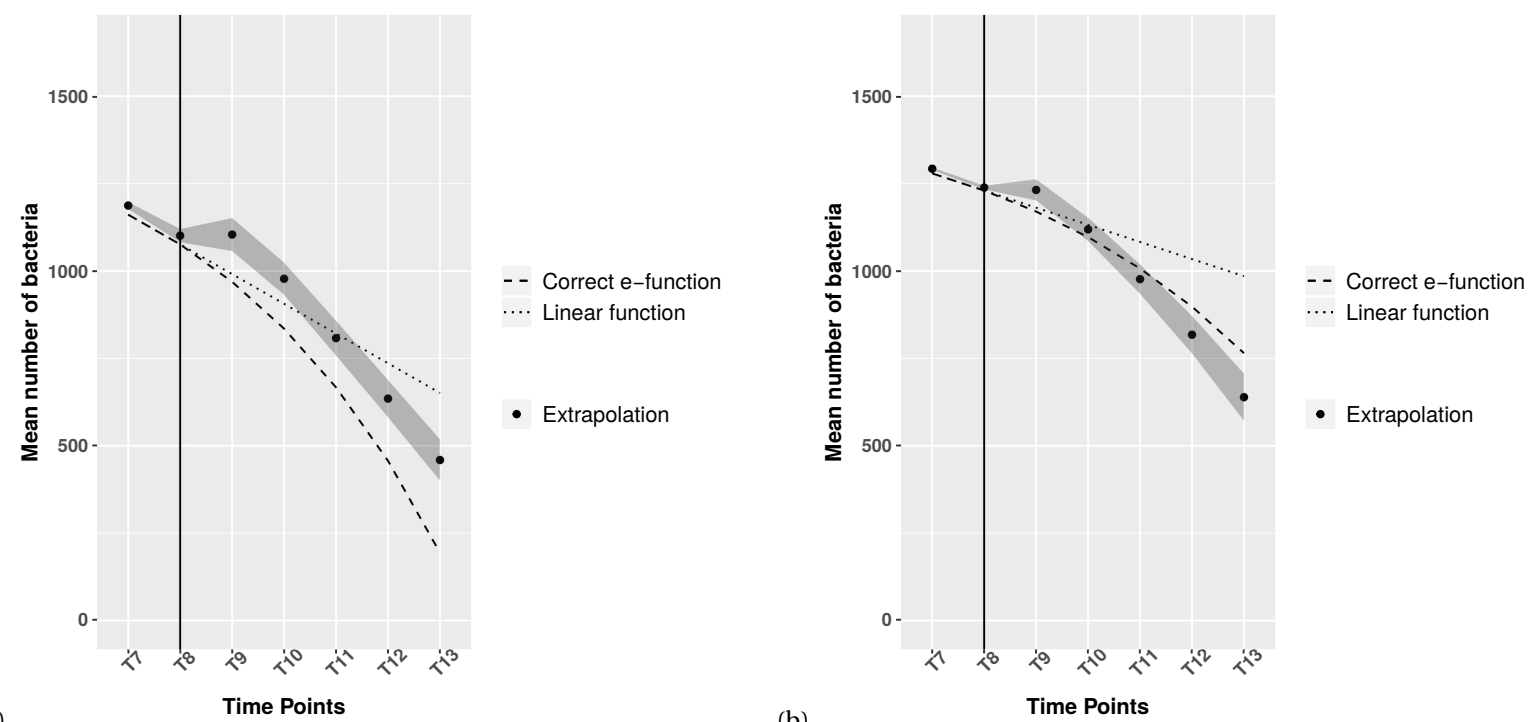

(a)

(b)

Fig. 4 Mean number of bacteria for Ain and Bin for first derivatives approach. The figure displays the mean number of bacteria estimated by participants in the grid condition who were classified as having abstracted a rule about the underlying process for (a) Ain and (b) Bin by calculating the first derivatives.

(2) Least squares approach. Using the least squares approach outlined above, $52 \%$ of participants were classified as having understood the correct function shape for process Ain, and 59\% for process Bin (Figure 5). These results are broadly in line with results using the derivatives approach.

Proportion of rule-based learners in all three conditions. Table 7 shows the proportion of participants classified as having acquired an understanding of the function rule for both processes, per condition. Across the two function-learning conditions, standard and summary, 19\% of participants were classified as rule-based learners for process Ain and $32 \%$ for process Bin. In contrast, in the grid condition 50\% of participants were classified as rule-based learners for Ain and 57\% for Bin.

Table 7 Proportion of participants classified as "rule-based learners" in all three conditions.

\begin{tabular}{cccccccc}
\hline \hline & Standard FL & Summary FL & $\begin{array}{l}\text { (a) Total: Stan- } \\
\text { dard \& Summary }\end{array}$ & Derivatives & $\begin{array}{l}\text { Least- } \\
\text { Squares }\end{array}$ & $\begin{array}{l}\text { (b) } \\
\text { Derivatives \& } \\
\text { Least-Squares }\end{array}$ & $\begin{array}{l}\text { Meanparison of } \\
\text { proportions of } \\
\text { (a) \& (b) }\end{array}$ \\
\hline Ain & $42(17 \%)$ & $50(20 \%)$ & $92(\mathbf{1 9 \%})$ & $80(48 \%)$ & $86(52 \%)$ & $166(\mathbf{5 0})$ & $\chi^{2}(1)=91.20^{* * *}$ \\
Bin & $82(33 \%)$ & $76(31 \%)$ & $158(\mathbf{3 2 \%})$ & $93(56 \%)$ & $97(59 \%)$ & $190(\mathbf{5 7 \%})$ & $\chi^{2}(1)=74.28^{* * *}$ \\
\hline
\end{tabular}

Comparison of proportions (a) FL and (b) grid condition, ${ }^{* * *} p \leq .001$. 


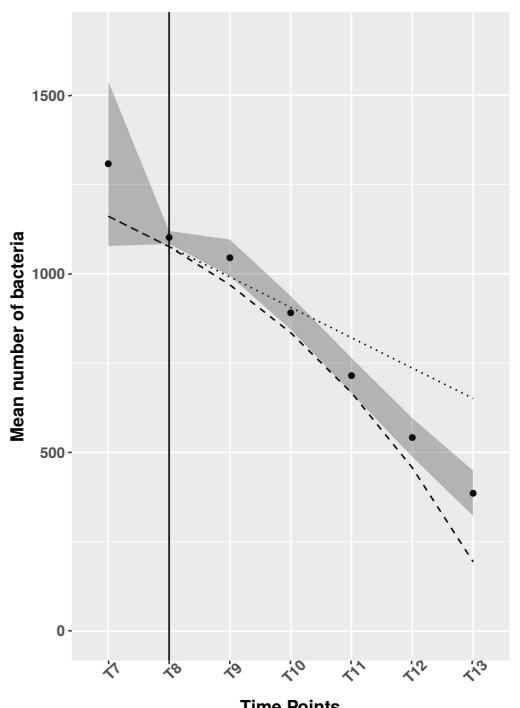

(a)

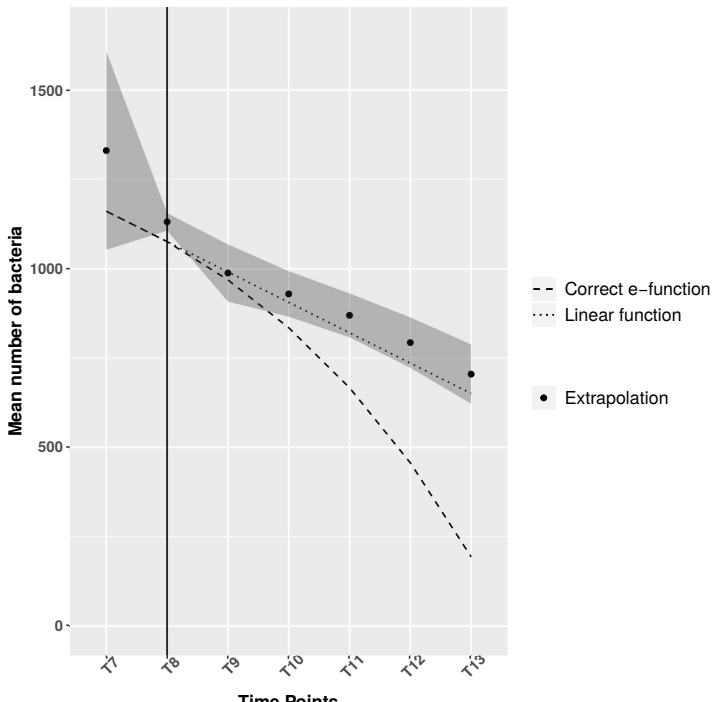

- Correct e-function

.... Linear function

- Extrapolation

Extrapolation (a)

Fig. 5 Mean number of bacteria for Ain and Bin for least squares approach. The figure displays the mean number of bacteria estimated by participants in the grid condition who were classified as having abstracted a rule about the underlying process for (a) Ain and (b) Bin applying a least squares approach. Pictures (c) and (d) display participants who were classified as extrapolating linearly.

\section{Summary 2}

Experiment 2 investigated whether unequal a priori probabilities to guess correctly could explain increased rule-learning compared to rule application in the standard function-learning paradigm. To do so, we introduced a condition where participants drew their understanding of the progress of the 
function into a grid where the numbers of clicks as well as the range of possible values were restricted to the same values as extrapolations in the standard function-learning task. The function shapes were evaluated using two different types of approaches, calculating the first derivatives, and a least squares approach of varying strictness. Both methods produced broadly similar results in that $50 \%$ of participants were classified as rule-based learners for process Ain and $57 \%$ for processes Bin, compared to $19 \%$ of participants showing rule-based extrapolation in both function-learning conditions for process Ain, and 32\% for process Bin.

To furthermore investigate whether (accurate) rule-application is reduced in the standard functionlearning paradigm because participants lack access to all previous function values, we compared extrapolation accuracy in the standard function-learning, with a summary function-learning condition. Results showed that there was no difference in extrapolation accuracy between the two conditions, suggesting that lacking access to all function values did not affect rule-application.

\section{General Discussion}

In the function-learning paradigm, people's understanding of the function rule that underlies the tobe extrapolated process is typically measured by means of extrapolation accuracy (DeLosh et al., 1997; Bott and Heit, 2004; Lewandowsky et al., 2002; McDaniel et al., 2014; Kwantes and Neal, 2006). Here we argue, however, that even though accurate extrapolations necessitate rule-learning, the reverse does not necessarily hold: Inaccurate extrapolations do not exclude rule-learning. Using inaccurate extrapolations to infer learning styles therefore hinges upon the assumption of rule-application given rule-learning. In two function-learning experiments with exponential declining functions, we put this assumption to an experiment test. Results showed that the proportion of participants who demonstrated an understanding of the correct function rule was almost twice as high as the proportion of participants who would be classified as rule-learners based on extrapolation accuracy in the standard function-learning experiment. We therefore conclude that (i) using extrapolation accuracy as a proxy for rule-learning severely underestimates people's actual ability to abstract the correct function rule; and that (ii) for a substantial proportion of participants, the assumption of rule-application given rule-learning does not hold. 
A majority of participants who would be classified as "exemplar-based learners" (61\%) or "simple exemplar-based learners" (46\%) based on their extrapolation accuracy, were able to identify the correct function shape in the rule-selection paradigm, and 37\% of the "exemplar-based learners" were able to identify the correct function shape AND slope. The grid paradigm that ensured equal a priori probabilities between drawing one's understanding of the rule and extrapolations in the classical function-learning paradigm produced broadly similar results: Around half of participants showed an accurate understanding of the function rule, both when analyzing their understanding of the exponentiality of the process via the first derivatives, and via a least squares approach. These results therefore suggest that a substantial proportion of participants did not apply their rule-understanding when extrapolating. In other words, extrapolation accuracy was considerably lower than what would be expected based on participants' understanding of the function rule.

In the grid paradigm, we employed different criteria varying in strictness of what counts as understanding of the function rule as exponential declining. Specifically, in the derivatives approach we varied the number of slopes that had to decrease strictly monotonically. It is important to note that even though strictly decreasing slopes are a characteristic feature of exponential declining functions, other non-linear functions could also meet this condition.

When the first and last slopes, as well as three out of four slopes were strictly monotonically decreasing, the proportion of rule-learners was higher than the proportion of rule-based extrapolators. Only when using the strictest criterion for rule-understanding, that all four slopes be strictly monotonically decreasing, the proportion of participants who were classified as having understood the rule was broadly in line with the proportion of participants who were classified as rule-based learners based on their extrapolation accuracy. This suggests that a considerable proportion of participants who had acquired an understanding of a characteristic feature of the function rule, namely that later extrapolation points should be steeper than earlier extrapolation points, could not implement this understanding when extrapolating.

Contrary to our expectation, extrapolation accuracy was not affected by implementation errors caused by a critical feature of classical function-learning experiments: the successive (as opposed to instantaneous) presentation of function values. Extrapolation accuracy was not higher in an alternative presentation format (summary function learning condition) that provided participants instantaneous access to current, as well as previous function values. This result suggests that while cognitive 
resources (working memory capacity) may be a limiting factor for rule-induction (McDaniel et al., 2014), they seem to be less relevant for rule-application during extrapolation.

Interestingly, while performance generally dropped as a function of slope, extrapolation accuracy was more strongly affected by function slope ( $>80 \%$ drop for Cin compared to Ain and Bin) compared to learning of the correct function shape (approx. 15\% drop for Cin compared to Ain and Bin). Furthermore, learning of the correct function shape AND slope was not influenced by function slope, suggesting that function slope impairs rule learning to a lesser extent than extrapolation accuracy. This result suggests that the well-established tendency toward linear extrapolations (Busemeyer et al., 1997) more strongly reflects a difficulty to extrapolate non-linearly than a more basic difficulty to recognize non-linear processes as non-linear.

The absolute minority of participants were classified as rule-based learners based on extrapolation accuracy. This pattern holds for both experiments, and for all functions slopes, except for one notable exception: In Experiment 2, about one-third of participants were classified as using rule-based extrapolation. That is, for the function with the less steepest slope, the highest proportion of rule-based extrapolators was reached. In line with this finding, previous research has repeatedly found that people expect linearly increasing function types (Busemeyer et al., 1997; Brehmer, 1974). The generally high proportions of participants who could identify the correct shape and slope of exponentially decreasing functions is therefore particularly telling in the present experiment using exponentially declining functions since these are among the function types with the strongest deviation from participants' expectation of positive linearity.

For participants displaying exemplar-based or simple exemplar-based extrapolations, the relative majorities (61\% and $46 \%$, respectively) could identify the correct function shape in the rule-selection task, while considerably smaller proportions of participants ( $24 \%$ and $32 \%$, respectively) believed the trained functions to be actually linear. That is, approximately half of participants extrapolating linearly were well-aware that extrapolations should not in fact be linear. For participants displaying rule-based extrapolations, the pattern was reversed in that the majority believed their extrapolation style to be accurate. These result suggest that participants, up to a certain extent, are aware of what accurate extrapolations should look like, and that around half of exemplar-based and simple exemplar-based extrapolators employed linear extrapolations despite their understanding of non-linearity. 
It is a common assumption of many function-learning studies that given that participants acquired an understanding of the function rule, they also apply that rule during extrapolation. The present results suggest, however, that a considerable proportion of participants who had acquired an understanding of the accurate function shape, and even slope displayed exemplar-based or even simple exemplar-based extrapolation in the classical function-learning paradigm. We conclude that rule-learning is not tantamount to rule application and that the proportion of rule-based learners in the current function-learning literature likely represents an underestimation.

Acknowledgements The authors gratefully acknowledge support by the Excellence Initiative, Institutional Strategy ZUK 5.4 (Scientific Computing in the Social and Behavioral Sciences), Heidelberg University.

All data and the analysis scripts (R) are stored under https://figshare.com/s/6be582d99deee687c126. (The data \& scripts will be uploaded before publication. )

\section{References}

Bott L, Heit E (2004) Nonmonotonic extrapolation in function learning. Journal of Experimental Psychology: Learning, Memory, and Cognition 30(1):38

Brehmer B (1974) Hypotheses about relations between scaled variables in the learning of probabilistic inference tasks. Organizational Behavior and Human Performance 11(1):1-27

Busemeyer JR, Byun E, Delosh EL, McDaniel MA (1997) Learning functional relations based on experience with input-output pairs by humans and artificial neural networks. Studies in Cognition Knowledge, Concepts and Categories pp 408-437

Carroll JD (1963) Functional learning: The learning of continuous functional mappings relating stimulus and response continua. ETS Research Bulletin Series 1963(2):i-144

DeLosh EL, Busemeyer JR, McDaniel MA (1997) Extrapolation: The sine qua non for abstraction in function learning. Journal of Experimental Psychology: Learning, Memory, and Cognition 23(4):968

Fischer H, Holt DV (2016) When high working memory capacity is and is not beneficial for predicting nonlinear processes. Memory \& Cognition 45(3):404-412

Kalish ML, Lewandowsky S, Kruschke JK (2004) Population of linear experts: knowledge partitioning and function learning. Psychological Review 111(4):1072 
Kwantes PJ, Neal A (2006) Why people underestimate y when extrapolating in linear functions. Journal of Experimental Psychology: Learning, Memory, and Cognition 32(5):1019-1030

Lewandowsky S, Kalish M, Ngang SK (2002) Simplified learning in complex situations: Knowledge partitioning in function learning. Journal of Experimental Psychology: General 131(2):163

MacKinnon AJ, Wearing AJ (1991) Feedback and the forecasting of exponential change. Acta Psychologica 76(2):177-191

Mcdaniel MA, Busemeyer JR (2005) The conceptual basis of function learning and extrapolation: Comparison of rule-based and associative-based models. Psychonomic bulletin \& review 12(1):24-42

McDaniel MA, Cahill MJ, Robbins M, Wiener C (2014) Individual differences in learning and transfer: Stable tendencies for learning exemplars versus abstracting rules. Journal of Experimental Psychology: General 143(2):668 